\title{
Visualização de Modelos Digitais: Informação dos Edifícios em Canteiro de Obras
}

\author{
Substituição da documentação impressa por digital
}

\author{
Mikael Brochardt \\ UFPE, Brazil \\ kall_muller@outlook.com \\ Max Andrade \\ UFPE, Brazil \\ maxandrade@uol.com.br
}

Jonas Assis

UFPE, Brazil

jonas.henrique@ufpe.br

\begin{abstract}
The use of mobile devices has brought important alternatives for various sectors, especially productive. Thus, studies and testing of these technologies has been used in the construction of buildings, that was not fully spread throughout the world, especially in developing countries, despite showing great potential for growth itself. Even with appropriate difficulties, the scenario for its implementation is positive, since it is certain diffusion, leaving thereby specular when these methods may be used without restriction, moreover, the use of BIM like a productivity gain factor accompanies such developments.
\end{abstract}

Keywords: BIM; Visualization; Information; Application; Productivity.

\section{Introdução}

Atualmente, no setor da construção civil, podemos destacar a busca pelo aproveitamento de tempo e mão-de-obra, visando reduzir custos de forma eficiente e regular. O ponto mais importante dessa observação é de como isso pode ser feito, e o que torna a resposta ainda mais complexa é, que existem várias formas para que isso ocorra como desejado, porém, integrar todos os setores de forma aplicada ao objetivo, gerando uma interdependência de um único sistema gerenciador é a forma mais plausível de realização. Mesmo sabendo disso, alguns setores do sistema tradicional de construção civil não são notados, pois seu custo de manutenção é visto apenas como investimento necessário sem que haja uma reavaliação de valores e métodos alternativos que permitam o pleno funcionamento do setor.

Como uma forma alternativa de gerenciamento de projetos desde o conceber até a manutenção pós entrega, pode-se destacar o Building Information Modeling (BIM), pois permite potencializar o fluxo das informações do edifício e permite armazenar as informações sobre os componentes da construção (Goes e Santos, 2011), tendo grande potencial para incrementar a produtividade na construção civil. As ferramentas de visualização e os métodos de compatibilização de sistemas prediais atreladas ao BIM têm mostrado como meios eficazes de redução de erros de projeto que levam a erros construtivos e retrabalhos. A possibilidade de agregar todos os sistemas prediais em um único modelo completo, por exemplo, oferece vantagens consideráveis reduzindo as perdas da informação no canteiro de obras e possibilitando a identificação de conflitos antecipadamente à execução da obra. Apesar desse potencial, a utilização dos modelos BIM para os canteiros de obras ainda é incipiente. Grande parte do uso do BIM ainda se dá durante as etapas de projeto e compatibilização dos modelos do edifício. Mesmo aqueles projetos que têm sido desenvolvidos em softwares de autoria BIM, na prática a informação chega ao canteiro de obras por meio de plantas impressas. Essas além de conterem informações abstratas, muitas vezes, difíceis de entender, ainda são incompletas, factíveis de erros e difíceis de serem alteradas. Mesmo ao se trabalhar com modelos BIM complexos e ricos de informação, ao transformar a documentação em papel impresso, muitas das informações se "perdem".

Hoje pode-se contar com o potencial do BIM adaptado para dispositivos portáteis a um nível satisfatório, com avançadas ferramentas de simulação, layers, especificação de materiais, balões informativos, plantas, cortes em tempo real, fachadas, cobertas, detalhamento de instalações elétricas e hidráulicas, permitindo até um tratamento de renderização para apresentações mais gerais. Isso permite resolver problemas rotineiros relacionados a documentação em todas as etapas da edificação, do projeto preliminar, à manutenção após a entrega, atuando eficazmente nos problemas mais complexos. Conjectura-se, que a substituição da representação impressa por uma representação digital, vinculada ao modelo BIM, em canteiros de obras permita facilitar 0 acesso à documentação, reduzir erros de informação, corrigir alterações de maneira mais automatizada, atualizar os modelos automáticos, contribuindo com a redução do desperdício em aumento da produtividade.

Além do mais, a substituição da documentação em papel por uma documentação digital resulta numa melhoria da qualidade ambiental do projeto. Durante o desenvolvimento da obra, o uso de documentos impressos aparece já na fundação do edifício do edifício e vai até o acabamento e até o projeto "como construído" (as build). Somadas às cópias de cada revisão do mesmo projeto a quantidade de recursos 
renováveis utilizada no canteiro de obras é significativamente grande.

\section{Objetivos}

É dentro desse contexto que a presente pesquisa se insere. $\mathrm{O}$ objeto de estudo desta pesquisa é o fluxo de informação de projeto nas etapas de construção de edifícios multifamiliares em altura, em um modelo comparativo que permita uma reflexão sobre a implementação de documentação digital aliada a plataforma BIM.

Um outro ponto é de propor uma revisão do sistema atual de fluxo da informação durante as fases da edificação, com o foco nas fases finais da construção de edifícios multifamiliares em altura, com a substituição da documentação impressa por documentos digitais, passíveis de serem utilizados em tablets e smartphones. Entre os projetos propostos no estudo destacam-se os projetos de arquitetura, estrutura, instalações hidro sanitárias, instalações elétricas e instalações especiais. A ênfase do projeto em que esse estudo se propôs pretende estudar nesta pesquisa é a fase de acabamento das unidades habitacionais por representar maior deficiência e carência de novos métodos.

Como objetivo específico, propõe-se a criação de métodos alternativos de visualização de projeto, modelados em softwares de autoria BIM; mostrar como esses métodos podem contribuir com a prática construtiva e com a melhoria dos processos de trabalho no canteiro de obras, melhorando o fluxo da informação e reduzindo os retrabalhos, onde, aliados podem representar economia e fluidez num setor burocrático e financeiramente pesado.

Não faz parte do objetivo desse trabalho citar as construtoras ou edificações estudadas por razões éticas. A relação do estudo e as empresas privadas é de soma de fatos junto a conhecimento científico, onde as dificuldades são notadas e sentidas por ambos, setor privado e academia. Também houve uma relação de gentileza com os proprietários em relação as informações cedidas, e falhas nominalmente relacionadas aos seus imóveis os prejudicaria.

\section{Implicações de pranchas físicas digitais}

\section{Fluxo de documentação em pranchas físicas}

O tempo gasto com a localização do documento desejado, dificuldade de manuseio devido as dimensões da prancha, o desgaste do material em virtude da fragilidade do papel, a multiplicidade de interpretação pela imprecisão das informações bidimensionais são alguns dos problemas corriqueiros da informação impressa presente nos canteiros de obras.

Hoje no Brasil, gestos consideravelmente comuns de consulta a informações, necessárias para a execução de determinada atividade, são sinônimos de transtornos e desgaste, sendo em alguns casos, capaz de mobilizar múltiplos funcionários. Isso ocorre por um conjunto de fatores, erroneamente vistos como normais no canteiro de obras, pois, frequentemente perde-se a impressão desejada meio a outras milhares de cópias arquivadas, devido a mudanças da localização de determinado setor que contém tal informação, relação desproporcional de organização/demanda de documentos, excesso de burocratização do manuseio dos arquivos, fluxo devotado entre vários indivíduos, ou ainda, quando se encontra o documento, o mesmo não está apto pois o desgaste natural do papel ou os dejetos produzido pelo próprio canteiro dificulta sua leitura.

Subsetores de projeto em especial, são capazes de maximizar tais falhas de forma expressiva. Hoje no Brasil, as grandes construtoras de produtos residenciais de médio e alto padrão possuem o setor de reformas e personalização, buscando oferecer além do produto padrão, a opção de adaptar e personalizar o imóvel utilizando métodos de estrutura independente e planta livre com suas devidas limitações. Nessa opção, o cliente, munido do projeto padrão, contrata a construtora ou um arquiteto de sua preferência para fazer as alterações necessárias (esse novo projeto pode ser digital ou não), o projeto retorna ao escritório para devida aprovação, após o aceite, uma cópia da pasta contendo suas informações

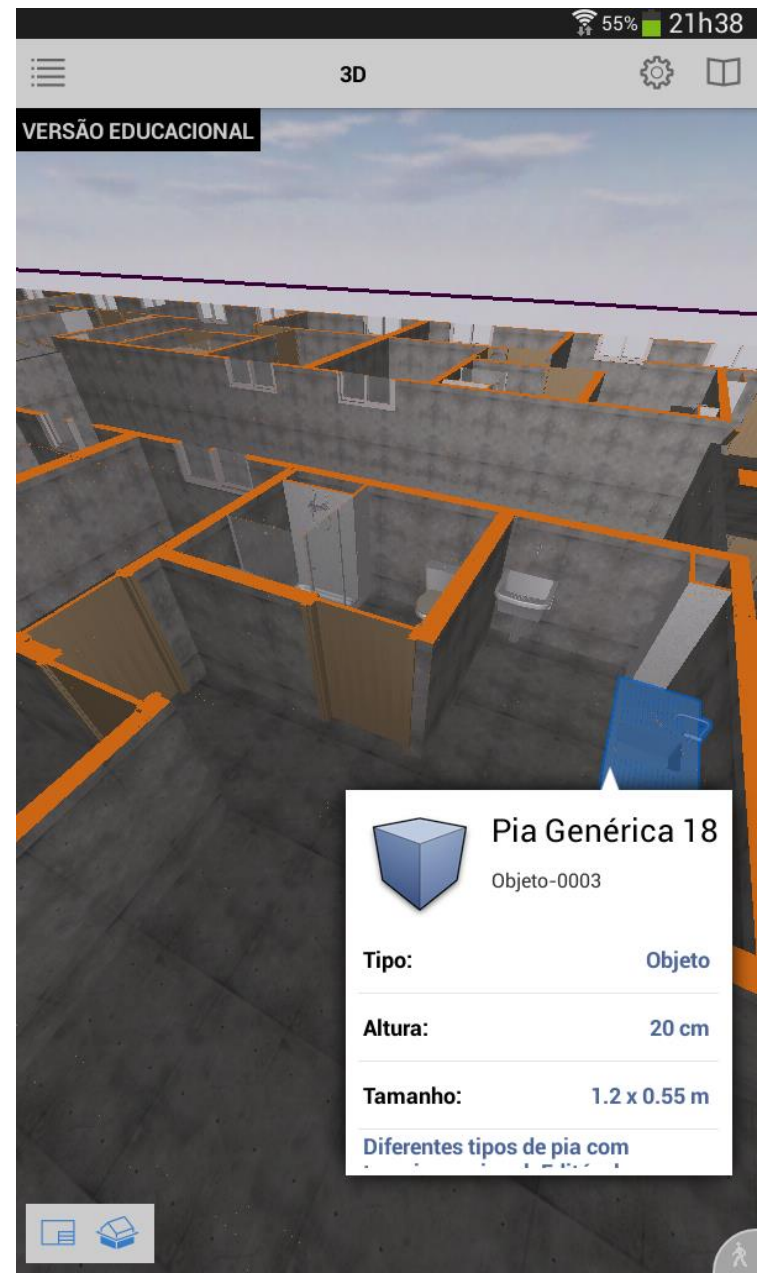

Imagem 1: Visualização de corte tridimensional no BIMx com destaque para consulta de informações sobre os elementos. 
segue para o canteiro. Cada projeto desse possui inúmeras pranchas, além disso, contam com várias revisões, aumentando a necessidade de um sistema eficaz de armazenamento e visualização de projeto.

\section{Fluxo de documentação em pranchas digitais}

Por outro lado, o uso das tecnologias digitais aliadas ao conceito BIM aparece como uma alternativa eficiente de substituição das documentações tracionais. Com a utilização de novas formas de visualização e documentação do edifício é possível se ter uma maior compreensão dos edifícios virtuais, reduzindo conflitos, retrabalhos e permitindo uma maior compreensão do edifício, em suas múltiplas faces.

O método de armazenamento digital não necessita de grandes espaços e, não sofre aumento com a chegada de novos projetos como é o caso das pranchas físicas, também, é possível tornar o fluxo desses projetos mais dinâmico utilizando conexão via internet e uma base de dados no escritório por exemplo, onde, qualquer um que necessite e tenha autorização para visualizar um único arquivo simultaneamente poderá fazê-lo. Além disso, poderão receber atualizações do arquivo em questão de forma rápida, onde poderiam haver várias fontes simultâneas, como por exemplo, revisões no projeto hidráulico e de revestimento, sendo operado por dois setores diferentes de projeto, mas em acordo, corrigindo determinado erro que envolve ambos os setores, assim, a tomada de decisão no canteiro é muito mais rápida e determinante.

Existem alguns visualizadores específicos para esse tipo de trabalho, como os tablets industriais, com maior resistência a impactos e com satisfatório poder de processamento. Esses aparelhos têm o custo um pouco mais elevado se comparado aos aparelhos comuns do mercado, porém, representam maior segurança ao investimento, principalmente onde sinistros são mais frequentes. Em uma obra pequena ou em fase de acabamento, aparelhos comumente encontrados no mercado, podem ser usados sem grandes riscos. Em relação a softwares, é necessário criar aplicativos que gerenciem de forma total cada fase dessa documentação, porém, já é possível gerenciá-los com mais de um aplicativo, onde cada um possui uma função. Alguns aplicativos já têm seu uso popularizado com interfaces intuitivas e amigáveis, como o BIMx (BIM), AutoCad 360 (Cad), além de aplicativos de análise de projeto como o Tekla BIMsight e o Field 3d.

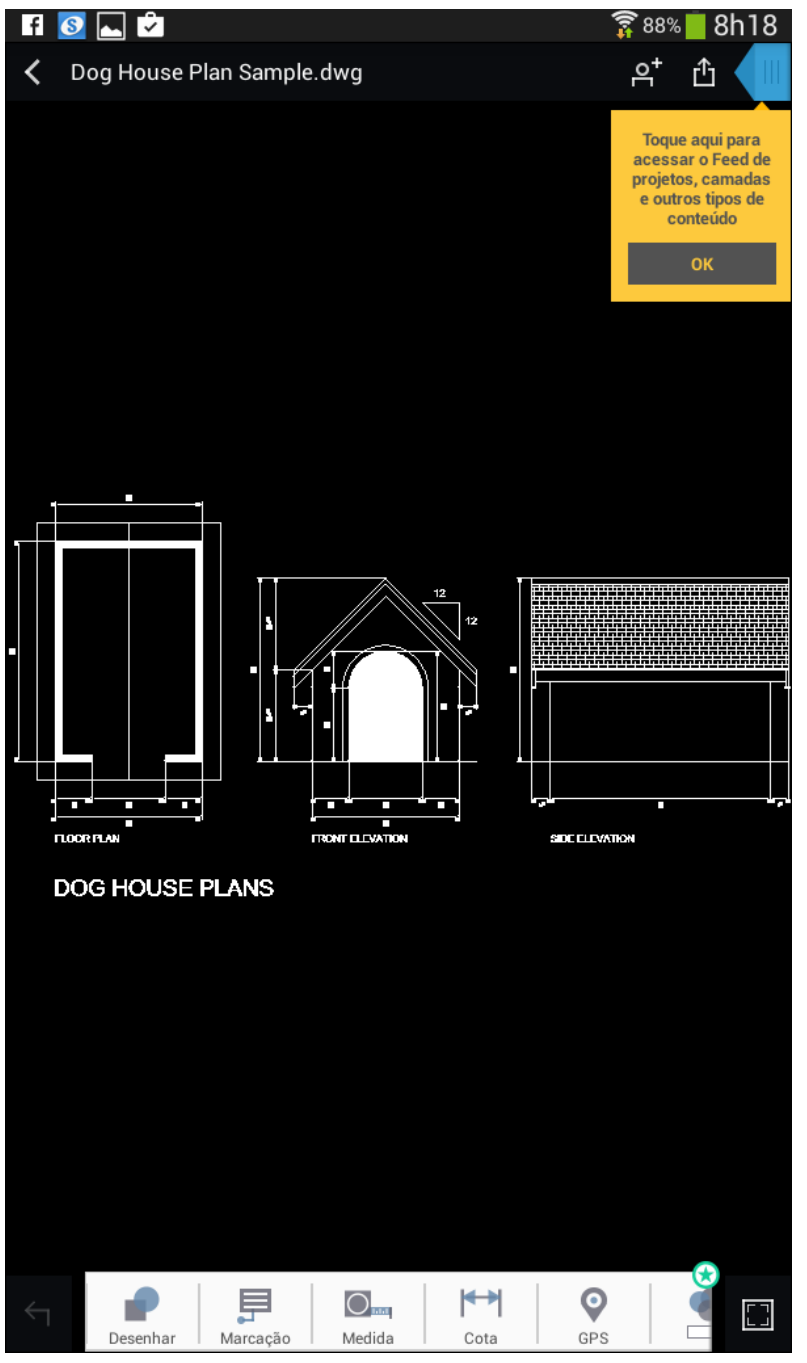

Imagem 2: Ambiente de visualização do AutoCAD 360, com destaque para as instruções mostradas nas primeiras utilizações.

As principais dificuldades para implementação desse tipo de recurso são o tempo de adaptação ao novo método, custos com treinamento e o investimento inicial, que se compararmos a impressões tradicionais possui o custo elevado, pois as impressões são efetuadas paulatinamente, apenas quando necessárias. Porém, o principal fator a ser levado em conta principalmente pelas grandes construtoras é o custo total, que considerando apenas impressões, o método digital já se mostra vantajoso, mesmo desconsiderando fatores importantes como redução de retrabalho, redução de custos de logística, tempo levado para a informação chegar ao canteiro, clareza de informações, mobilidade no canteiro e vários outros.

\section{Simulação}

Por tratar de tecnologias recentes, existem poucas avaliações práticas em relação as suas características financeiras, uma das razões para que as informações sobre elas fiquem rapidamente ultrapassadas é a frequência em que novas tecnologias de hardware chegam ao mercado, o que barateia 
as anteriores, além de novas aplicações mostrando-se cada vez mais intuitivas e otimizadas. Nesse estudo, será feita uma análise de valores superficiais de equipamentos para que seja instaurada a documentação digital. Tais valores são referentes a média de custo da região metropolitana do Recife, Brasil, onde os valores serão dados na moeda local $(R \$)$. Tal consulta foi feita entre o mês de junho e julho de 2016.

Também, é importante ressaltar que a avaliação será baseada em uma obra residencial de médio/alto padrão da cidade em questão, onde, e o setor será o de reformas e personalização, devido a sua carência de soluções para documentação. Assim, consideremos 20, a média de revisões dos projetos, levando em conta as regras padrão para aprovação do que foi solicitado, excluindo detalhes mínimos como altura corrigida de ponto elétrico, assim temos por revisão:

- Dois cortes do pavimento ou apartamento requerido;

- Uma prancha de demolição;

- Prancha de construção;

- Projeto hidráulico;

- Projeto Elétrico;

- Projeto de instalações de gás;

- $\quad$ Projeto de revestimento;

- Elevação do revestimento.

Após listados os itens básicos, vamos considerar que cada ponto seja impresso em formato A1 (NBR 10068) expresso em cores ( $R \$ 5,00$ cada prancha), logo, observando a média de valor de tais impressões nas datas e local já citados, temos:

\begin{tabular}{|lccc|}
\hline Prancha & Qnt/Proj. & № de revisões & $\begin{array}{c}\text { Impressões } \\
\mathrm{A} 1(\mathrm{R} \$)\end{array}$ \\
\hline Cortes & 2 & 20 & 200,00 \\
\hline Demolição & 1 & 20 & 100,00 \\
\hline Construção & 1 & 20 & 100,00 \\
\hline Hidráulica & 1 & 20 & 100,00 \\
\hline Elétrica & 1 & 20 & 100,00 \\
\hline Gás & 1 & 20 & 100,00 \\
\hline Revestimento & 1 & 20 & 100,00 \\
\hline Elev. Revestimento & 1 & 20 & 100,00 \\
\hline & \multicolumn{3}{c}{ Total R\$ } \\
\hline
\end{tabular}

Sabendo que, o total de impressões para a realização das revisões resultou no total de $R \$ 900,00$ reais e, considerando que esse conjunto de revisões devem ser enviadas como cópias para os interessados e responsáveis, levaremos em conta o setor de reformas no escritório, o canteiro de obras para execução, cliente ou arquiteto responsável, assim, temos um custo de $R \$ 2.700,00(900,00 \times 3)$ por projeto de reforma realizado apenas com impressão. Devemos lembrar que, esse valor é referente a apenas um apartamento e, traçando novamente uma média, podemos obter em um edifício com 60 apartamentos, aproximadamente 20 unidades com projetos de reforma, assim, chegamos à $\mathrm{R} \$ 54.000,00$ (2.700 x 20), considerando apenas a documentação de projeto do setor. Em uma contagem mais expressiva, todos os setores deverão ser contabilizados, assim como outros fatores de ganho de produtividade relacionados ao uso de documentação digital e o BIM.

\section{Fundamentação teórica}

\section{Ganho de produtividade na plataforma BIM}

Segundo Picchi (1993), o desenvolvimento de várias etapas de projetos em plataformas diferentes, deixa lacunas de informação entre os produtos adquiridos, o que certamente compromete a ideia inicial do edifício, desfigurando em alguns aspectos, o produto resultante. As divergências de informações são causadas pelo caminhar paralelo entre as etapas de projeto, comprometendo a qualidade do produto, causada pela incompatibilidade resultante.

A substituição das representações bidimensionais por modelos tridimensionais do edifício traz uma série de vantagens, mas, sem a integração das informações do edifício pode gerar abstrações simbólicas bidimensionais, como as plantas e os cortes (GOES \& SANTOS, 2011 apud. FERREIRA, 2007). Essa forma abstrata de compreender e compatibilizar o projeto tem se mostrado insatisfatória, contribuindo com a geração de erros e retrabalhos na fase de execução. Para Goes e Santos (2011), o modelo BIM, ao permitir a criação de um protótipo virtual do edifício, permite armazenar todas as informações necessárias acerca de todos os sistemas do prédio. Nas ferramentas de autoria BIM é possível a geração automática de plantas, cortes e fachadas, utilizando-se as simbologias técnicas exigidas pelas normas brasileiras, a partir dos tridimensionais (GOES \& SANTOS, 2011). Dessa forma, o processo de modelagem em BIM, por ser mais complexo e preciso, acaba por demandar mais tempo. Porém, o modelo gerado contém todos as informações outrora distribuídos em vários desenhos bidimensionais contendo projetos de hidráulica, estrutura, elétrica, etc. A unificação dos projetos em um modelo único permite a identificação e solução de conflitos durante o próprio processo de modelagem, além de permitir a utilização de ferramentas de compatibilização, como o Tekla BIMSight.

Portanto, a compatibilização adequada do projeto, especialmente a que utiliza o BIM se mostra um fator importante de redução de erros na construção do edifício. Ainda assim, apesar de apresentar maior coerência e qualidade de compatibilização em relação aos desenhos que são realizados em ferramentas CAD, os desenhos gerados a partir do modelo BIM para uso na obra ainda são abstrações simbólicas bidimensionais, que desperdiçam parte da riqueza semântica do modelo BIM. Para Souza, "a tecnologia está sendo usada mais como ferramenta de concepção e como facilitadora do processo de projeto dentro do escritório de arquitetura, não atingindo diretamente outros processos ligados à produção do edifício". (SOUZA, 2009)

Uma das soluções de inserção da informação de modo mais completo no canteiro de obras é a utilização de tablets e smartphones, dispensando a impressão das plantas. A permanência no meio digital mesmo durante a fase executiva propicia um melhor aproveitamento das informações do 
modelo BIM, permitindo a visualização de mais desenhos sem custos adicionais e visualização tridimensional. Permite também isolar e cruzar as informações desejadas dos vários elementos e sistemas que compõem o edifício, propiciando riqueza e clareza de informação, além de facilitar e agilizar o gerenciamento de plantas. Os dispositivos móveis apresentam como grande vantagem em relação a plantas impressas ou monitores fixos a mobilidade de locomoção no canteiro. Amaral destaca também a mobilidade de dados possibilitada por esses aparelhos, que são capazes de sincronizar e atualizar informações projetuais através da internet, melhorando o fluxo de informações entre o escritório de projeto e o canteiro de obras (AMARAL, 2012).

\section{DISCUSSÃO}

Acredita-se que a permanência da informação no meio digital, mesmo durante a fase executiva da obra propicia um melhor aproveitamento das informações do modelo BIM, permitindo a extração completa e precisa da informação do edifício, sem a necessidade de uso de desenhos abstratos e conflitantes, além de evitar os gastos corriqueiros de impressão de projetos.

O emprego de dispositivos móveis tem como grande vantagem em relação às plantas impressas ou monitores fixos, a mobilidade de locomoção no canteiro e o baixo custo de investimento (em longo prazo). Efetivamente melhorando o fluxo de informações entre o escritório de projeto e melhorar a qualidade da informação no canteiro de obras. Mas, para isso, a identificação dos principais aplicativos existentes no mercado, o conhecimento dos seus potenciais e suas características deve ser o primeiro passo de um processo de substituição dos desenhos impressos no canteiro de obras por informações digitais oriundos de aplicativos para smartphones e tablets.

\section{Referências}

AMARAL, Mariane C.; DUARTE, Glaucius D. DESENVOLVIMENTO DE UM APLICATIVO WEB PARA GERENCIAMENTO DE OBRAS DE CONSTRUÇÃO CIVIL PARA USO EM TABLETS. UFPEL, Pelotas-RS, 2012.

BUENO, A. R.;MORAES, A. S. S. As ferramentas do planejamento em obras civis como mecanismo de redução de custos e aumento da produtividade. Trabalho de Conclusão de Curso, Universidade da Amazônia, UNAMA. Belém-PA, 2010.

EASTMAN, Chuck; TEICHOLZ, Paul; SACKS, Rafel e LISTON, Kathleen. - BIM Handbook - A Guide to Building Information Modeling. Second Edition. New Jersey, John Wiley \& Sons, Inc, 2011.

FABRICIO, Márcio Minto; MELHADO, Silvio Burrattino. Por um processo de projetosimultâneo. In: II WORKSHOP NACIONAL: GESTÃO DO PROCESSO DEPROJETO NA CONSTRUÇÃO DE EDIFÍCIOS, 2002, Porto Alegre.

GOES, B.; SANTOS, E. T.; TONISSI, R. H. Compatibilização de projetos: comparação entre o BIM e o CAD2D. TIC 2011 Encontro de Tecnologias de Informação e Comunicação na Construção, FAUFBA, Salvador-BA, 2011

KOSKELA, Lauri. 1992. Process Improvement and Automation in Construction: Opposing or Complementing Approaches? The 9th International Symposium on Automation and Robotics in Construction, 3 - 5 June 1992, Tokyo. Proceedings.

PICCHI, F.A. Sistemas da Qualidade: Uso em Empresas de Construção. São Paulo, 1993. 462p. Tese (Doutorado) - USP

SOUSA, O. K.; MEIRIÑO, M. J. Aspectos da implantação de ferramentas BIM em empresas de projetos relacionados à construção civil.Artigo aceito em 2013, pelo IX Congresso Nacional de Excelência em Gestão.

SOUZA, L. A.; AMORIM, S. L.; LYRIO, A. M. Impactos do uso do bim em escritórios de Arquitetura: Oportunidades no mercado imobiliário. Gestão \& Tecnologia de Projetos (Periódico Científico do Instituto de Arquitetura e Urbanismo da USP), SP, 2009. 